\title{
Glucose administration attenuates hypothermia-induced retrograde amnesia in rats in a time- and dose-dependent manner
}

\author{
ROBERT W. FLINT, JR., and DAVID C. RICCIO \\ Kent State University, Kent, Ohio
}

\begin{abstract}
It is well established that posttraining administrations of glucose enhance memory in a time- and dose-dependent manner. More recently, glucose has also been found to attenuate scopolamine-induced anterograde amnesia, displaying the same time- and dose-response characteristics (Stone et al., 1988). In the present study, the use of glucose as an agent for alleviation of hypothermia-induced retrograde amnesia was examined. In each experiment, animals were given a single trial of passive avoidance conditioning. Retrograde amnesia was induced through hypothermia immediately following training. All injections were given immediately following hypothermia treatment, and tests for fear retention were administered $24 \mathrm{~h}$ following training. In Experiment 1, the dose-response effects of glucose $(10,100$, or $400 \mathrm{mg} / \mathrm{kg}$ ) were examined; in Experiment 2, the time-dependent effect of $100 \mathrm{mg} / \mathrm{kg}$ of glucose administered immediately, 1 , or $2 \mathrm{~h}$ after hypothermia was examined. The results show that glucose injections attenuate retrograde amnesia in both a dose- and time-dependent manner. These results coincide with the growing body of evidence supporting the memory-modulating effects of glucose.
\end{abstract}

In the last two decades, a large body of evidence has accumulated suggesting that glucose may be involved in modulating memory storage and retrieval in animals (see Gold, 1991, 1992, 1995, for reviews). Early beliefs about the mechanisms through which glucose affected memory focused around its association with epinephrine. Gold and van Buskirk (1978) discovered that posttraining injections of epinephrine could enhance performance of a passive avoidance task in rats in an inverted-U dose-response curve and in a time-dependent manner. However, epinephrine does not easily cross the blood-brain barrier into the central nervous system (Axelrod, Weil-Malherbe, \& Tomchick, 1959), suggesting that its effects must be through some peripheral mechanism. Epinephrine causes an increase in the amount of circulating blood glucose, which does cross the blood-brain barrier (Ellis, Kennedy, Eusebi, \& Vincent, 1967 ) and may be responsible for the effects of epinephrine on memory.

Although the exact mechanisms through which glucose affects memory have not yet been determined, evidence supporting both peripheral (Messier \& White, 1987; Rodriguez, Horne, Mondragon, \& Phelps, 1994; White, 1991; Williams \& Jensen, 1991; Williams \& McGaugh, 1992) and central (Gold, 1995; Lee, Graham, \& Gold, 1988) mechanisms of action have been extensively discussed.

Gold (1995) has recently suggested that while peripheral administration of glucose as well as other hormones and

This research was supported in part by National Institute of Mental Health Grant MH37535 to D.C.R. Care of animals was in accordance with Kent State University guidelines. Correspondence regarding this article should be directed to R. W. Flint, Jr., Department of Psychology, Kent State University, Kent, OH 44242-0001 (e-mail: rflint@kentvm. kent.edu). neurotransmitter-related drugs have memory-enhancing properties for a wide variety of tasks, central administration of these agents into specific brain targets and brain lesions of the amygdala and medial septum indicate that these task effects are anatomically specific. One interpretation is that because peripheral injections are not anatomically specific they influence many brain structures, including the ones involved in the task at hand, thus accounting for the broad effects of glucose. However, when the injections are controlled centrally, the task must be sensitive to the anatomical location of the injection.

Despite the lack of a neurological mechanism of action, behavioral studies of glucose have identified its common characteristics on memory. Glucose has specific time- and dose-dependent qualities with respect to its effects on the target memory. Gold (1986) first found this effect with glucose by showing that a range of doses $(1.0-500 \mathrm{mg} / \mathrm{kg})$ administered immediately following passive avoidance training yielded an inverted- $U$ dose-response curve. Furthermore, the optimal dose in that study, $100 \mathrm{mg} / \mathrm{kg}$, was ineffective if administered $1 \mathrm{~h}$ following training.

Behavioral studies have documented these characteristic effects of glucose in a wide variety of areas. Specifically, the memory effects of glucose have been widely studied in normal rats and mice using both aversive (see Gold, 1991, for a review) and appetitive (see White, 1991, for a review) paradigms. Glucose has also been shown to attenuate agerelated deficits (Sternberg, Martinez, Gold, \& McGaugh, $1985)$ and to alleviate disruptions in circadian rhythms (Stone, Rudd, Ragozzino, \& Gold, 1992), conditioned suppression (Messier \& White, 1987), and working memory (Ahlers, Shurtleff, Schrot, Thomas, \& Paul-Emile, 1993). In humans, glucose has been shown to enhance memory in studies with normal young adults (Hall, Gonder-Frederick, 
Chewning, Silveira, \& Gold, 1989) as well as with elderly patients with age-related memory deficits (Parsons \& Gold, 1992) in both an anterograde and retrograde manner (Manning, Parsons, \& Gold, 1992). Furthermore, word recognition, recall, orientation, face recognition, and narrative prose have all shown improvement as a result of glucose administration in patients with senile dementia of the Alzheimer's type (Manning, Ragozzino, \& Gold, 1993). Thus, it appears that glucose has memory-enhancing properties for a broad range of different tasks from normal rats to humans with Alzheimer's dementia.

One area of research that appears to have been neglected with respect to the retrograde effects of glucose on memory is that of experimentally induced amnesia. Stone, Croul, and Gold (1988) examined the effects of glucose on scopolamine-induced anterograde amnesia in rats on performance of an inhibitory avoidance task. They found that administration of a $100-\mathrm{mg} / \mathrm{kg}$ dose of glucose immediately following training significantly attenuated the amnestic effects of the minimum effective dose of scopolamine. However, if 10 or $250 \mathrm{mg} / \mathrm{kg}$ was used, or if $100 \mathrm{mg} / \mathrm{kg}$ was administered $1 \mathrm{~h}$ after training, glucose had no effect. Their findings, using an anterograde amnesia paradigm, yielded the same dose-response curve and timedependent characteristics previously associated with glucose in other studies.

Whether the effects of glucose on amnesia has generality for other paradigms, such as retrograde amnesia induced through means other than direct pharmacological manipulations, is not yet known. Accordingly, the present study sought to extend the findings of Stone et al. (1988) by investigating the dose- and time-dependent effects of glucose on hypothermia-induced retrograde amnesia (Riccio, Hodges, \& Randall, 1968) for passive avoidance training in rats.

\section{GENERAL METHOD}

\begin{abstract}
Animals and Housing
The animals were Long-Evans female rats from the breeding colony at Kent State University. They were housed individually on a 15:09-h light:dark schedule with Purina Rat Chow and water available ad lib. All training took place during the light phase of the light:dark schedule.
\end{abstract}

\footnotetext{
Apparatus

The passive avoidance apparatus was a $33 \times 17 \times 17 \mathrm{~cm}$ chamber constructed of Plexiglas and steel, and divided into two equal-sized compartments separated by a guillotine door. In one compartment, the walls were constructed of white translucent Plexiglas and the lid was clear Plexiglas. The other compartment was made entirely of black opaque Plexiglas. The dividing wall and guillotine door $(10 \times 8 \mathrm{~cm})$ were constructed so that each side was consistent with the color of the chamber it faced. The floor in each compartment was constructed of stainless steel bars, $2 \mathrm{~mm}$ in diameter and spaced $1 \mathrm{~cm}$ apart. A 3-sec $125-\mathrm{V}$ unscrambled shock from a matched impedance ac shock source could be administered in the black side. The room was illuminated with a $15-\mathrm{W}$ light bulb suspended above the white chamber, and the experiment was conducted in the presence of white noise to mask background disturbances. A stopwatch was used to measure the animals' crossover latencies and total time on the white side.

Hypothermia was induced by using a Model 1095 Forma Temp Jr. water bath and circulator, which was maintained at $3^{\circ} \pm 0.5^{\circ} \mathrm{C}$. Subjects receiving hypothermia treatment were restrained in clear
}

Plexiglas tubes and immersed up to their necks until their colonic temperatures were lowered to $22.0^{\circ} \mathrm{C}$. A rectal probe inserted approximately $2.5 \mathrm{~cm}$ into the rat's anus was used to measure colonic body temperature.

\section{Procedure}

All subjects were handled for $3-5 \mathrm{~min}$ each day for 2 days prior to training. Training involved taking each subject into the experimental room and holding it for $10 \mathrm{sec}$ while it became acclimated to its surroundings. The animal was then placed in the white compartment for $10 \mathrm{sec}$ before the guillotine door was raised. The latency for all four feet to cross to the black compartment was measured. If the animal did not cross in $60 \mathrm{sec}$, it was removed from the experiment. After the rat crossed to the black compartment, the door was immediately lowered and the rat was given a $3-\mathrm{sec} 125-\mathrm{V}$ inescapable shock. Subjects that received hypothermia treatment were immediately removed and exposed to the cold bath described above. Subjects in the control groups that did not receive hypothermia treatment were simply removed from the training apparatus and returned to their home cages. Injections of glucose or saline were administered subcutaneously in the back of the neck. Glucose was obtained from the Sigma Chemical Company.

Twenty-four hours after hypothermia, each animal was returned to the white compartment of the testing apparatus to be tested for retention of passive avoidance training. When the guillotine door was raised, the initial crossover latency was recorded along with the total time spent in the white compartment (TTW) as measures of retention. No shock was administered during testing.

\section{EXPERIMENT 1}

Experiment 1 was designed to determine whether the effects of glucose administered immediately following hypothermia treatment were dose dependent. The invertedU-shaped dose-response curve for memory enhancement through glucose administration has become an identifying characteristic of its effect on memory. On the basis of the results of prior research, three doses of glucose were selected $(10,100$, and $400 \mathrm{mg} / \mathrm{kg})$. It was suggested that the moderate dose might attenuate hypothermia-induced retrograde amnesia, whereas small and large doses would probably be less effective.

\section{Method}

Thirty-nine rats (190-265 g) were divided randomly into five groups. Four groups received subcutaneous injections immediately following hypothermia treatment; three of these groups received glucose at $10 \mathrm{mg} / \mathrm{kg}(n=6), 100 \mathrm{mg} / \mathrm{kg}(n=7)$, or $400 \mathrm{mg} / \mathrm{kg}$ $(n=6)$, and one group received saline $(n=8)$. To ensure that learning had occurred, a retention control group $(n=7)$, which did not receive the hypothermia treatment, was also included. Five animals died during the experiment due to experimenter error. A $300-\mathrm{sec}$ retention test was administered $24 \mathrm{~h}$ after training.

\section{Results and Discussion}

Initial crossover latencies from training were compared using a Kruskal-Wallis one-way analysis of variance (ANOVA). The results indicated that no significant differences existed among the groups prior to testing $(H=2.02$, $p>.05$ ). A Kruskal-Wallis one-way ANOVA on both latencies (LAT) and TTW scores yielded significant group differences (LAT, $H=15.02, p<.05$; TTW, $H=20.83, p<$ .05). All subsequent pairwise comparisons were performed using nonparametric two-tailed Mann-Whitney $U$ tests. Both the LAT and TTW scores are presented in Figure 1 
(panels A and B, respectively). Within each group, latency and TTW scores were similar except for the $400-\mathrm{mg} / \mathrm{kg}$ glucose group. The discrepancy between LAT and TTW scores for the $400-\mathrm{mg} / \mathrm{kg}$ group will be discussed later in detail.

Scores for the no-hypothermia control group were significantly higher than those of the saline-hypothermia control group (LAT, $U=7, p<.05$; TTW, $U=4, p<.01$ ), indicating that retrograde amnesia had been induced. The LAT and TTW scores in Figure 1 both show the characteristic inverted-U dose-response curve typically associated with posttraining glucose administration. Comparison of the scores for the saline control with the various levels of glucose administration indicated comparable performance in the $10-\mathrm{mg} / \mathrm{kg}$ dose (LAT, $U=21.5, p>.5$; TTW, $U=19, p>.5$ ) and significant differences for both the $100-\mathrm{mg} / \mathrm{kg}$ (LAT, $U=6, p<.05$; TTW, $U=3, p<$ .01 ) and $400-\mathrm{mg} / \mathrm{kg}$ doses (LAT, see below; TTW, $U=5$, $p<.05$ ). These results indicate that, as with the saline group, $10 \mathrm{mg} / \mathrm{kg}$ of glucose was ineffective at attenuating the amnestic effects of severe hypothermia. However, both
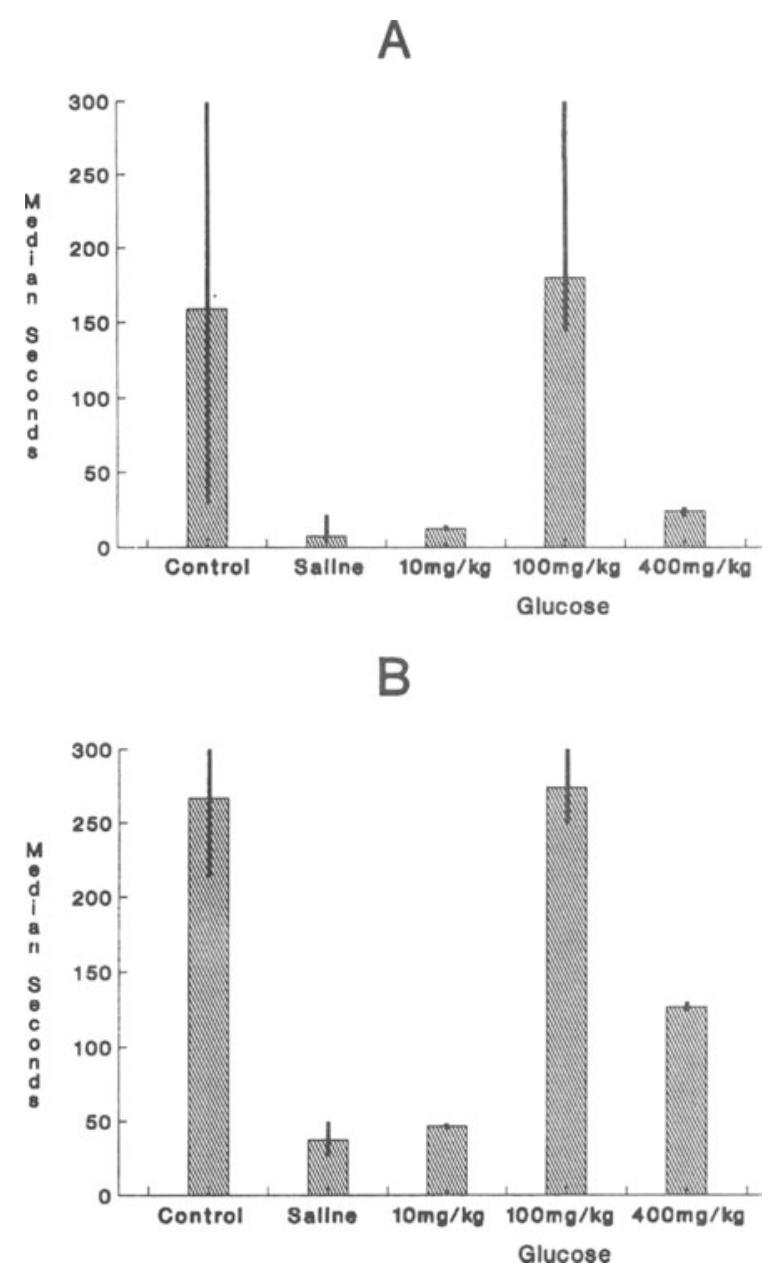

Figure 1. (A) Median latency and (B) total time on white for the control, saline, $10-\mathrm{mg} / \mathrm{kg}$ glucose, $100-\mathrm{mg} / \mathrm{kg}$ glucose, and 400-mg/kg glucose groups. Error bars $=$ semi-interquartile range. the $100-\mathrm{mg} / \mathrm{kg}$ dose and the $400-\mathrm{mg} / \mathrm{kg}$ dose were sufficient to alleviate the hypothermic effects on memory.

This finding was further borne out with comparisons among the treatment groups. The TTW scores for the $10-\mathrm{mg} / \mathrm{kg}$ group differed significantly from those for both the 100 - and $400-\mathrm{mg} / \mathrm{kg}$ groups (TTW, $U=1$ and $U=2$, respectively, $p<.05$ ), again indicating the effectiveness of the larger doses at attenuating the amnestic effects of hypothermia. Furthermore, the $100-$ and $400-\mathrm{mg} / \mathrm{kg}$ groups did not differ significantly (TTW, $U=9.5, p>.05$ ). The same pattern of results was found in each comparison using the LAT scores, except that there was no difference between the 10- and 400-mg/kg groups (LAT, $U=6.5, p>.05$ ).

There was an additional mild inconsistency in the pattern of data for the LAT and TTW scores with respect to the saline control and the 400-mg/kg glucose groups. Although the analysis did not show any significant difference between the groups for LAT $(U=10.5, p>.05)$, the TTW comparison did yield a significant difference $(U=5, p<.05)$. One explanation for this difference in dependent variables may be the type of behavior reflected in each of these measures. Occasionally subjects cross over relatively quickly, when given the opportunity to do so, only to return and spend most of their time in the white compartment (i.e., low LAT and high TTW). In these cases, a number of possibilities could explain their behavior; the animals might have been momentarily disinhibited as a result of the guillotine door's being raised, or the animals may have failed to initially attend to their surrounding contextual cues, or the cues on the black side may have effectively reactivated the memory. Each of these factors might have contributed to the differences we found between our dependent measures. It is for this reason that we recorded both LAT and TTW for each animal and why we often consider TTW a more sensitive measure of retention.

\section{EXPERIMENT 2}

In the second experiment, the time-dependent effects of glucose in attenuating retrograde amnesia were investigated using the effective dose of $100 \mathrm{mg} / \mathrm{kg}$ from Experiment 1 . Immediately following a single trial of passive avoidance training, subjects in the amnestic groups were given hypothermia treatment to induce amnesia. To examine the effects of glucose, the subjects were given injections of glucose $(100 \mathrm{mg} / \mathrm{kg})$ or saline immediately or $1 \mathrm{~h}$ or $2 \mathrm{~h}$ following the hypothermia treatment.

\footnotetext{
Method

The subjects were 70 rats $(225-360 \mathrm{~g})$, which were divided into six groups. Five of the groups received hypothermia immediately following training. Three of these groups received s.c. injections of glucose $(100 \mathrm{mg} / \mathrm{kg})$ immediately $(n=15)$ or $1 \mathrm{~h}(n=9)$ or $2 \mathrm{~h}$ $(n=9)$ following hypothermia treatment. As there was no reason to expect a time-dependent effect of saline, only immediate $(n=14)$ and $1-\mathrm{h}(n=10)$ saline groups were included. The sixth group, a retention control group $(n=10)$ that did not receive the amnestic treatment, was employed to ensure that initial learning of the task had occurred. Three animals were excluded because they did not meet the training criterion or because of experimenter error.
} 


\section{Results and Discussion}

Initial crossover latencies from training were compared using a Kruskal-Wallis one-way ANOVA. The results indicated that no significant differences existed among any of the groups before testing $(H=5.70, p>.05)$. A KruskalWallis one-way ANOVA showed a significant treatment effect for both LAT and TTW scores $(H=18.91, p<.05$, and $H=22.13, p<.05$, respectively). All subsequent pairwise comparisons were performed using nonparametric two-tailed Mann-Whitney $U$ tests. Data for both the LAT and TTW scores yielded similar results for all groups.

Subjects in the no-hypothermia retention control group displayed good retention by remaining on the white (safe) side of the apparatus. In contrast, both of the hypothermia saline control groups displayed poor memory of the training task by spending relatively little time on the white side. Furthermore, the immediate and $1-\mathrm{h}$ saline groups did not differ statistically (LAT, $U=54.5, p>.1$; TTW, $U=$ $55, p>.1)$ and were combined for subsequent statistical analysis. The differences in performance between the nohypothermia control group and the combined saline group indicated that hypothermia treatment effectively induced retrograde amnesia (see Figure 2). Results for the glucosetreatment groups varied as a function of the delay interval. Subjects that received glucose immediately or $1 \mathrm{~h}$ following hypothermia showed good retention, whereas subjects that received glucose $2 \mathrm{~h}$ following hypothermia displayed substantial amnesia.

These impressions were confirmed by the statistical analysis. Comparing the scores for the no-hypothermia control group with the scores for the saline condition (combined immediate saline and 1-h saline groups) indicated that hypothermia induced retrograde amnesia (LAT, $U=$ $47.5, p<.01$; TTW, $U=37.5, p<.01$ ). There was a significant improvement in performance when the saline condition was compared with glucose when administered immediately (LAT, $U=70, p<.01$; TTW, $U=55, p<.001$ ) or $1 \mathrm{~h}$ (LAT, $U=59.5, p<.05$; TTW, $U=53.5, p<.05$ ) following hypothermia treatment. However, an analysis of the 2-h-delay group did not reveal any significant differences in relation to the saline condition (LAT, $U=89.5$, $p>.1$; TTW, $U=105, p>.5$ ).

These findings indicated that $100 \mathrm{mg} / \mathrm{kg}$ of glucose attenuated retrograde amnesia in a time-dependent manner: Amnesia is eliminated or weakened if glucose is administered within $1 \mathrm{~h}$ following the hypothermia treatment, but remains strong if glucose administration is delayed for $2 \mathrm{~h}$.

\section{GENERAL DISCUSSION}

The results of these two experiments support and extend the growing body of evidence that glucose can have memory-modulating properties. In general, a moderate $(100 \mathrm{mg} / \mathrm{kg})$ dose of glucose clearly resulted in substantial attenuation of retrograde amnesia. However, a low $(10 \mathrm{mg} / \mathrm{kg})$ dose of glucose was ineffective in attenuating hypothermia-induced amnesia, and a high ( $400 \mathrm{mg} / \mathrm{kg})$ dose produced either weak or no attenuation, depending on the particular measure. Furthermore, the $100-\mathrm{mg} / \mathrm{kg}$ dose of
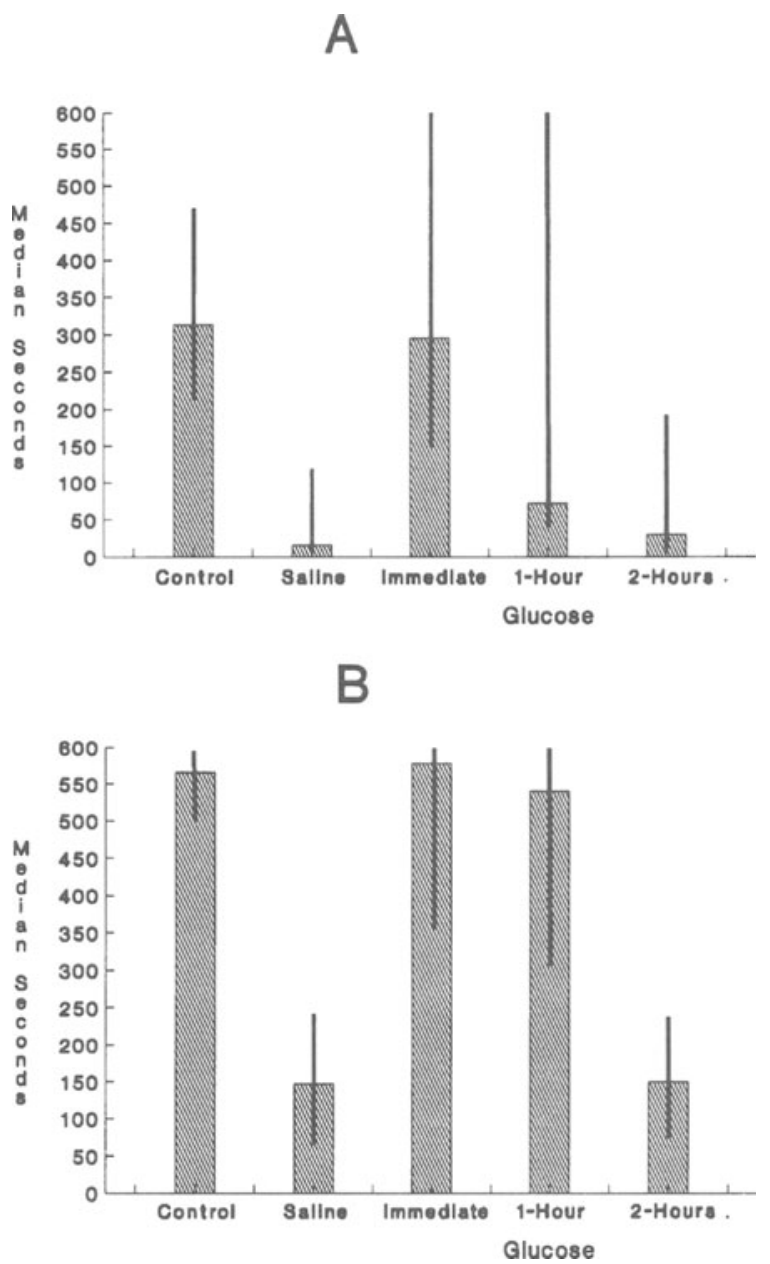

Figure 2. (A) Median latency and (B) total time on white for the control, combined immediate and 1-h delay saline, immediate glucose, 1-h delay glucose, and 2-h delay glucose groups. Error bars $=$ semi-interquartile range.

glucose was effective in attenuating the amnestic effects of hypothermia when administered within $1 \mathrm{~h}$ following treatment, but not if administered $2 \mathrm{~h}$ after hypothermia.

In general, these findings paralleled the characteristics associated with the memory-modulating effects of glucose found repeatedly in prior studies (Gold, 1986, 1991, 1995). However, in the time-dependent study, the memorymodulating effects of glucose remained effective after a longer delay than previously reported.

It is unclear why glucose was effective after a longer delay, although there were a number of differences in both the paradigm and procedure, such as the use of hypothermia as the amnestic agent, the retrograde amnesia paradigm, and the sex of the animals. An additional possibility is that the effect of hypothermia was not complete after $1 \mathrm{~h}$ and the glucose offset its effect.

Although the present study was a behavioral investigation and physiological data were not obtained, results from other research may permit some speculation as to the neural processes underlying the effects of glucose on memory. It 
has been suggested that temperature changes in the hippocampus may account for cold-induced deficits in working memory (Ahlers, Thomas, \& Berkey, 1991) and that these deficits may be alleviated through glucose administration as a result of increased metabolism (Ahlers et al., 1993). If this were the case, an increase in glucose causing an increase in metabolism should offset subtle temperature changes in the hippocampus. Presumably, then, a $400-\mathrm{mg} / \mathrm{kg}$ dose would be more effective than a $100-\mathrm{mg} / \mathrm{kg}$ dose. However, we did not find evidence to support this suggestion.

It has also been reported that cold exposure can induce seizure activity (Gehres, Randall, Riccio, \& Vardaris, 1973), which causes a change in the neurotransmitters in the brain. Along the same line, Ahlers et al. (1991) have suggested that the memory deficits associated with cold exposure may be the result of changes in the neurotransmitter systems in, for example, the hippocampal cholinergic system, which is important for working memory. The results of the present study appear to fit the idea that hypothermia changes some neurotransmitter system in the brain. Thus, hypothermia-induced amnesia may have resulted in a change in the neurotransmitter system involving the amygdala, which is associated with emotional memories (LeDoux, 1993). The optimal dose of glucose presumably prevents this change caused by hypothermia and allows for the expression of the memory during later testing. However, if the optimal dose of glucose is not administered, hypothermia will disrupt the neurotransmitter system and cause amnesia at the time of testing.

This is the first study indicating that glucose can act to attenuate retrograde amnesia. Furthermore, the source of memory loss was a nonpharmacological manipulation involving hypothermia. These results fit nicely with prior research displaying similar inverted-U dose-response relationships and time-dependent relationships that have become characteristic of the memory-modulating effects of glucose.

\section{REFERENCES}

Ahlers, S. T., ShurtlefF, D., Schrot, J., Thomas, J. R., \& PaulEMILE, F. (1993). Glucose attenuates cold-induced impairment of delayed matching-to-sample performance in rats. Psychobiology, 21, 87-92.

Ahlers, S. T., Thomas, J. R., \& Berkey, D. L. (1991). Hippocampal and body temperature changes in rats during delayed matching-tosample performance in a cold environment. Physiology \& Behavior, 50, 1013-1018.

Axelrod, J., Weil-Malherbe, H., \& Tomchick, R. (1959). The physiological disposition of $3 \mathrm{H}$-epinephrine and its metabolite metanephrine. Journal of Pharmacology \& Experimental Therapeutics, 127, 251-256.

Ellis, S., Kennedy, B. L., Eusebi, A. J., \& Vincent, N. H. (1967). Autonomic control of metabolism. Annals of the New York Academy of Sciences, 139, 826-832.

Gehres, L. D., Randall, C., Riccio, D. C., \& Vardaris, R. M. (1973). Pharmacologic blockade of hypothermic retrograde amnesia. Physiological Psychology, 1, 973-976.

GoLD, P. E. (1986). Glucose modulation of memory storage processing. Behavioral \& Neural Biology, 45, 342-349.

GOLD, P. E. (1991). An integrated memory system: From blood to brain. In R. C. A. Frederickson, J. L. McGaugh, \& D. L. Felten (Eds.), Periph- eral signaling of the brain: Role in neural-immune interactions and learning and memory (pp. 391-419). Lewiston, NY: Hogrefe \& Huber. GoLD, P. E. (1992). Modulation of memory processing: Enhancement of memory in rodents and humans. In L. R. Squire \& N. Butters (Eds.), Neuropsychology of memory (pp. 402-414). New York: Guilford Press.

GoLD, P. E. (1995). Modulation of emotional and nonemotional memories: Same pharmacological systems, different neuroanatomical systems. In J. L. McGaugh, N. M. Weinberger, \& G. Lynch (Eds.), Brain and memory: Modulation and mediation of neuroplasticity (pp. 41-74). New York: Oxford University Press.

GolD, P. E., \& VAN BuskiRK, R. B. (1978). Effects of alpha- and betaadrenergic receptor antagonists on post-trial epinephrine modulation of memory: Relationship to post-training brain norepinephrine concentrations. Behavioral Biology, 24, 168-184.

Hall, J. L., Gonder-Frederick, L. A., Chewning, W. W., Silveira, J., \& GoLD, P. E. (1989). Glucose enhancement of performance on memory tests in young and aged humans. Neuropsychologia, 27, 1129-1138.

LeDoux, J. E. (1993). Emotional memory systems in the brain. Behavioral Brain Research, 58, 69-79.

LeE, M., Graham, S., \& Gold, P. E. (1988). Memory enhancement with post-training intraventricular glucose injections in rats. Behavioral Neuroscience, 102, 591-595.

Manning, C. A., Parsons, M. W., \& Gold, P. E. (1992). Anterograde and retrograde enhancement of 24 -h memory by glucose in elderly humans. Behavioral \& Neural Biology, 58, 125-130.

Manning, C. A., Ragozzino, M. E., \& Gold, P. E. (1993). Glucose enhancement of memory in patients with probable senile dementia of the Alzheimer's type. Neurobiology of Aging, 14, 523-528.

Messier, C., \& White, N. M. (1987). Memory improvement by glucose, fructose, and two glucose analogs: A possible effect on peripheral glucose transport. Behavioral \& Neural Biology, 48, 104-127.

Parsons, M. W., \& Gold, P. E. (1992). Glucose enhancement of memory in elderly humans: An inverted-U dose-response curve. Neurobiology of Aging, 13, 401-404.

Riccio, D. C., Hodges, L. A., \& Randal.L, P. K. (1968). Retrograde amnesia produced by hypothermia in rats. Journal of Comparative \& Physiological Psychology, 66, 61 8-622.

Rodriguez, W. A., Horne, C. A., Mondragon, A. N., \& Phelps, D. D. (1994). Comparable dose-response functions for the effects of glucose and fructose on memory. Behavioral \& Neural Biology, 61, 162-169.

Sternberg, D. B., Martinez, J., Gold, P. E., \& McGaugh, J. L. (1985). Age-related memory deficits in aged mice and rats: Enhancement with peripheral epinephrine. Behavioral \& Neural Biology, 44, 213-220.

Stone, W. S., Croul, C. E., \& Gold, P. E. (1988). Attenuation of scopolamine-induced amnesia in mice. Psychopharmacology, 96, 417-420.

Stone, W. S., Rudd, R. J., Ragozzino, M. E., \& Gold, P. E. (1992). Glucose attenuation of deficits in memory retrieval in altered light: dark cycles. Psychobiology, 20, 47-50.

WHITE, N. (1991). Peripheral and central memory-enhancing actions of glucose. In R. C. A. Frederickson, J. L. McGaugh, \& D. L. Felten (Eds.), Peripheral signaling of the brain: Role in neural-immune interactions and learning and memory (pp. 421-441). Lewiston, NY: Hogrefe \& Huber.

Williams, C. L., \& Jensen, R. A. (1991). Vagal afferents: A possible mechanism for the modulation of memory by peripherally acting agents. In R. C. A. Frederickson, J. L. McGaugh, \& D. L. Felden (Eds.), Peripheral signalling of the brain: Role in neural-immune interactions and learning and memory (pp. 467-472). Lewiston, NY: Hogrefe \& Huber.

Williams, C. L., \& McGaugh, J. L. (1992). Reversible inactivation of the nucleus of the solitary tract impairs retention performance in an inhibitory avoidance task. Behavioral \& Neural Biology, 58, 204-210.

(Manuscript received March 8, 1995; revision accepted for publication December 1, 1995.) 